\title{
ENSAYO FITOGEOGRÁFICO DE LA PTERIDOFLORA DEL CW HISPANO
}

\author{
Miguel Angel MARTÍN BALLESTEROS, Montserrat MARTÍNEZ ORTEGA, \\ $\mathrm{M}^{\mathrm{a}}$ José PÉREZ HORNERO y Enrique RICO HERNÁNDEZ
}

\begin{abstract}
RESUMEN. Ensayo fitogeográfico de la pteridoflora del CW hispano. El notable conocimiento de la distribución de los pteridófitos en el CW hispano (provincias administrativas de Zamora, Salamanca y Cáceres), ha servido como base para la aplicación de cuatro tipos de análisis que permiten valorar la importancia fitogeográfica de la pteridoflora en la zona: índice $\mathrm{m} / \mathrm{t}$, índice de similitud con las regiones próximas, semejanza entre provincias y sectores corológicos, y análisis histórico. Además se han distinguido algunos taxones indicadores biogeográficos.
\end{abstract}

Palabras clave. Pteridophyta, Fitogeografía, CW hispano.

ABSTRACT. Phytogeographical essay of CW Spanish pteridological flora. A good knowledge of the distribution of pteridophytes in CW Spain (Zamora, Salamanca and Cáceres provinces), is the base on we have applied four analyses that let us evaluate the phytogeographical importance of pteridophytes in this area: $\mathrm{m} / \mathrm{t}$ index, index of similarity with the adjacent regions, index of similarity between chorological divisions, and historical analysis. Finally, we have selected some taxa which have considered as biogeographical indicators.

Key words. Pteridophyta, Phytogeography, CW Spain.

\section{INTRODUCCIÓN}

La corología de los pteridófitos ibéricos ha sido objeto de varios estudios, tanto en trabajos que incluían el conjunto de la Península como en otros restringidos a áreas más puntuales. Los primeros (p.ej., Salvo et al., 1984) pusieron de manifiesto la necesidad de estudios pteridológicos más precisos, y así se llevaron a cabo algunos en diversas regiones: Andalucía (Salvo y Cabezudo, 1984 a y b), León (Díaz González y Puente García, 1985),
Navarra (Catalán y Aizpuru, 1988) y Valencia (Herrero Borgoñón et al., 1989). Nuestro trabajo se enmarca en esta necesidad de mejorar el conocimiento pteridoflorístico de la Península, ocupándonos de la región centro occidental hispana, que de una manera artificial hacemos coincidir con las provincias administrativas de Zamora, Salamanca y Cáceres. La elección de límites administrativos viene dada por la necesidad inicial de delimitar de forma precisa la zona que se va a herborizar y facilitar la recopilación de citas corológicas 
procedentes de bibliografía, dado que hasta tiempos recientes rara vez se han manejado unidades corológicas en publicaciones botánicas. Ciertamente el aprovechamiento de los datos no es todo lo amplio y fiable que se desearía, pues obviamente después, a la hora de hacer comparaciones en un ensayo fitogeográfico, debemos acudir a las unidades corológicas.

Tras herborizaciones continuadas durante varios años, especialmente entre 1991 y 1993 , hemos logrado un conocimiento lo suficientemente amplio de la pteridoflora del CW hispano como para acometer un estudio pormenorizado de las áreas de distribución de los taxones, así como un ensayo fitogeográfico de la pteridoflora, tomando como referencia trabajos de entre los que destacan los de Pichi Sermolli et al. (1988) y Salvo y García Verdugo (1990).

\section{METODOLOGÍA}

Previamente a este trabajo se ha confeccionado un atlas pteridológico de las provincias de Zamora, Salamanca y Cáceres. Para ello, en primer lugar, se llevó a cabo la revisión de material de herbarios, principalmente de los de las Facultades de Biología y Farmacia de la Universidad de Salamanca (herbarios SALA y SALAF, respectivamente), Real Jardín Botánico de Madrid (MA), y también, sobre todo para los casos de algunas citas bibliográficas, se consultó el herbario MAF (Facultad de Farmacia de la Universidad Complutense de Madrid).

Simultáneamente, se hizo una recopilación de citas bibliográficas y se comenzaron las campañas de herborización con el propósito de elevar el porcentaje de cuadrículas U.T.M. visitadas. Es necesario señalar que en los casos de especies que no presentan dificultades de identificación «de visu» en el campo, no siempre se han recolectado los ejemplares, con ánimo de preservar al máximo las poblaciones halladas.

Tras esta fase de recogida de datos, se empezó a elaborar un listado de todas las citas correspondientes a cada una de las especies, el cual ha servido como base para obtener el catálogo y atlas de los pteridófitos -en mapas del retículo UTM de $10 \times 10 \mathrm{~km}$ - de las provincias mencionadas, y una base de datos que facilitara el tratamiento informático de los mismos.

Sobre la base de dicho atlas, aplicamos diferentes tipos de análisis fitogeográficos, para los cuales se ha utilizado la división corológica expuesta por Rivas Martínez (1987), dentro de los límites políticos de las provincias mencionadas.

Las provincias y sectores corológicos representados dentro del área de estudio se muestran en la figura 1 y son los siguientes: la provincia Carpetano-Ibérico-Leonesa (CIL) que comprende los sectores: OrensanoSanabrense (OS), Lusitano-Duriense (LD), Salmantino (S); Bejarano-Gredense (BG), Leonés (L); la provincia CastellanoMaestrazgo-Manchega (CMM) con el sector Castellano-Duriense (CD); y la provincia LusoExtremadurense (LE) con un solo sector: el Toledano-Tagano (TT). A lo largo del texto para referirnos a cada unidad corológica se utilizarán las abreviaturas escritas entre paréntesis.

Se ha prescindido del sector Leonés en la mayoría de los análisis de este ensayo biogeográfico, dado que está muy poco representado en la zona estudiada y los datos que de él tenemos son insuficientes para establecer conclusiones fiables.

La frecuencia de aparición de cada taxón en cada sector corológico, que se recoge en la tabla 1, se ha establecido según la siguiente escala: -0-Ausente (no se ha encontrado en ese sector); -1- Raro (se conoce de menos del 10\% de las cuadrículas U.T.M. 10x10 del sector); - 


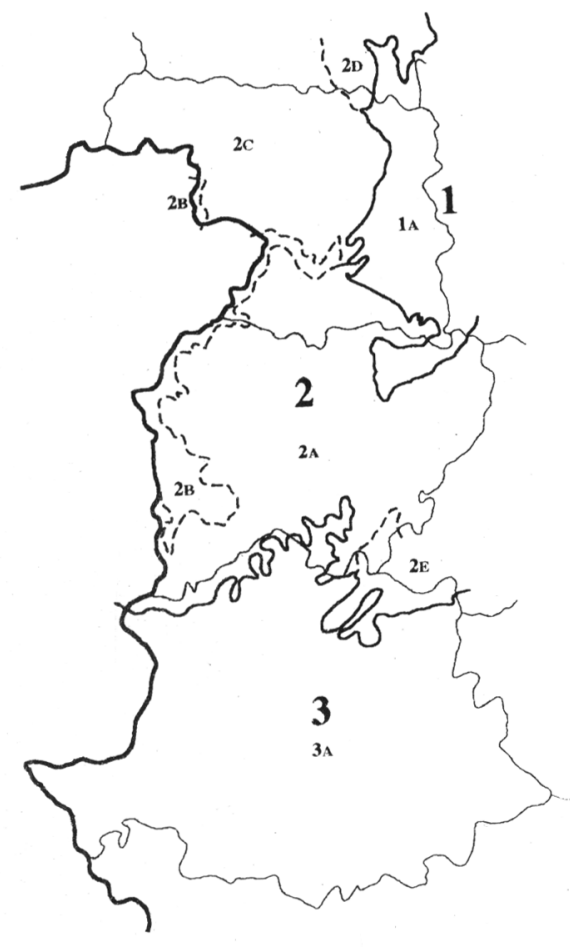

Figura 1. Provincias y sectores corológicos en Zamora, Salamanca y Cáceres. 1: CMM, 1A: CD. 2: CIL, 2A: S, 2B: LD, 2C: OS, 2D: L, 2E: BG. 3: LE, 3A: TT. Chorological division in Zamora, Salamanca and Cáceres. 1: CMM, 1A: CD. 2: CIL, 2A: S, 2B: LD, 2C: OS, 2D: L, 2E: BG. 3: LE, 3A: $T T$.

2- Ocasional (se conoce del 10-25\% de las cuadrículas); -3-Frecuente (se conoce de más del 25\% de las cuadrículas).

Para la división en regiones corológicas (Me: Mediterránea; Ma: Macaronésica y E: Eurosiberiana) y la presencia (+) o ausencia () de los taxones en ellas, que se recoge en las columnas "1" de la tabla 1, hemos seguido a Pichi Sermolli (1979).

En la columna "2" de esa tabla 1 se indican los taxones con esporas monoletas (m) o triletas (t), para obtener posteriormente el índice $\mathrm{m} / \mathrm{t}$. Siguiendo los trabajos de Itô $(1972,1978)$, el primero en aplicar el índice y obtener conclusiones del mismo, estableceremos seis rangos (A: valores mayores de 3; B: 2,51-3,00;
C: 2,01-2,50; D: 1,51-2,00; E: 1,01-1,50; F: menos de 1,00) (Figs. 3 y 4 ).

Los tipos biogeográficos se han establecido según lo expuesto en Pichi Sermolli et al. (1. c.). Los números de la columna "3" de la tabla 1 y los de la figura 10 corresponden a: TERCIARIO: circumboreal (1), heterocórico (2), mediterráneo y de otras regiones próximas (3), mediterráneo y de otras regiones orientales (4), submediterráneo (5), endemismo mediterráneo (6), relicto macaronésico (7). CUATERNARIO: circumboreal (8), heterocórico (9), mediterráneo y de otras regiones próximas (10).

A la hora de establecer la similitud entre las pteridofloras de dos unidades corológicas hemos utilizado el habitual índice de Sörensen (1948), que se obtiene por el cociente $Q=2 z /$ $(\mathrm{x}+\mathrm{y})$, donde $\mathbf{z}$ es el número de especies comunes entre las dos zonas a comparar; $\mathbf{x}$ el número total de especies de una zona; e y el número total de especies de la otra zona.

\section{RESULTADOS Y DISCUSIÓN}

Riqueza. Como primer resultado se muestra la tabla 1, que incluye los 58 taxones de pteridófitos conocidos en la actualidad en el CW hispano y los datos que corresponden a cada uno de ellos. Es importante reseñar que el sector leonés, del que prescindiremos en adelante, alberga la única población conocida hasta el momento de Equisetum palustre en el área de estudio.

Las áreas que presentan mayor densidad de taxones (fig. 2) son las más montañosas (Sierra Segundera, Sistema Central y Guadalupe-Villuercas), así como los encajonamientos del Duero en Las Arribes y, en menor medida, del Tajo en Monfragüe.

Por sectores biogeográficos, el que alberga mayor número de taxones es el ToledanoTagano, seguido por el Orensano-Sanabrense y el Salmantino. Es de destacar el bajo número 


\begin{tabular}{|c|c|c|c|c|c|c|c|c|c|c|c|c|}
\hline TAXONES: & OS & $\begin{array}{r}\mathrm{C} \\
\mathrm{LD}\end{array}$ & $\begin{array}{l}\text { I } \\
\text { S }\end{array}$ & $\begin{array}{c}\mathrm{L} \\
\mathrm{BG}\end{array}$ & $\mathrm{L}$ & \begin{tabular}{|l|} 
CMM \\
CD \\
\end{tabular} & $\begin{array}{l}\text { LE } \\
\text { TT }\end{array}$ & $\mathrm{Me}$ & $\begin{array}{l}1 \\
\mathrm{Ma}\end{array}$ & E & 2 & 3 \\
\hline Huperzia selago & 1 & 0 & 0 & 0 & 0 & 0 & 0 & - & + & + & & 7 \\
\hline Lycopodiella inundata & 1 & 0 & 1 & 0 & 0 & 0 & 0 & - & + & + & & 7 \\
\hline Selaginella denticulata & 0 & 0 & 0 & 0 & 0 & 0 & 2 & + & + & + & & \\
\hline Isoetes setaceum & 2 & 2 & 3 & 0 & 0 & 0 & 2 & + & - & + & & 5 \\
\hline Isoetes histrix & 2 & 3 & 3 & 1 & 0 & 0 & 3 & + & - & + & & 5 \\
\hline Isoetes velatum velatum & 2 & 2 & 1 & 1 & 0 & 0 & 1 & + & - & + & & \\
\hline Isoetes velatum asturicense & 1 & 0 & 1 & 2 & 0 & 0 & 1 & + & - & - & & ? \\
\hline Isoetes duriei & 0 & 1 & 1 & 0 & 0 & 0 & 1 & + & - & + & & ( \\
\hline Equisetum hyemale & 0 & 0 & 0 & 0 & 0 & 0 & 1 & + & - & + & & \\
\hline Equisetum ramosissimum & 2 & 2 & 2 & 0 & 2 & 2 & 1 & + & + & + & & \\
\hline Equisetum arvense & 1 & 2 & 1 & 0 & 2 & 0 & 1 & + & - & + & & \\
\hline Equisetum fluviatile & 1 & 0 & 0 & 0 & 0 & 0 & 0 & + & - & + & & \\
\hline Equisetum palustre & 0 & 0 & 0 & 0 & 2 & 0 & 0 & + & - & + & & \\
\hline Ophioglossum lusitanicum & 0 & 1 & 1 & 0 & 0 & 0 & 2 & + & + & + & $\mathrm{t}$ & \\
\hline Ophioglossum azoricum & 1 & 2 & 2 & 0 & 0 & 0 & 1 & + & + & + & $\mathrm{t}$ & \\
\hline Ophioglossum vulgatum & 1 & 1 & 1 & 1 & 0 & 1 & 1 & + & + & + & $\mathrm{t}$ & \\
\hline Osmunda regalis & 1 & 1 & 2 & 2 & 0 & 0 & 1 & + & + & + & $\mathrm{t}$ & \\
\hline Polypodium cambricum & 0 & 3 & 1 & 0 & 0 & 0 & 1 & + & - & + & $\mathrm{m}$ & \\
\hline Polypodium interjectum & 1 & 2 & 1 & 1 & 0 & 0 & 1 & + & - & + & $\mathrm{m}$ & \\
\hline Polypodium vulgare & 1 & 0 & 1 & 2 & 0 & 0 & 1 & + & - & + & $\mathrm{m}$ & \\
\hline Cheilanthes acrostica & 0 & 0 & 1 & 0 & 0 & 0 & 1 & + & - & + & $\mathrm{t}$ & \\
\hline Cheilanthes maderensis & 0 & 3 & 0 & 0 & 0 & 0 & 2 & + & + & - & $\mathrm{t}$ & \\
\hline Cheilanthes tinaei & 1 & 3 & 1 & 2 & 0 & 0 & 3 & + & + & - & $\mathrm{t}$ & \\
\hline Cheilanthes hispanica & 1 & 2 & 2 & 2 & 0 & 0 & 3 & + & - & + & $\mathrm{t}$ & \\
\hline Cryptogramma crispa & 2 & 0 & 0 & 3 & 0 & 0 & 0 & + & - & + & $\mathrm{t}$ & \\
\hline Adiantum capillus-veneris & 0 & 1 & 1 & 0 & 0 & 0 & 1 & + & + & + & $\mathrm{t}$ & \\
\hline Anogramma leptophylla & 1 & 3 & 2 & 1 & 0 & 0 & 3 & + & + & + & $\mathrm{t}$ & \\
\hline Cosentinia vellea & 0 & 1 & 0 & 0 & 0 & 0 & 1 & + & + & - & $\mathrm{t}$ & \\
\hline Marsilea strigosa & 1 & 0 & 1 & 0 & 0 & 0 & 1 & + & - & + & & \\
\hline Pilularia globulifera & 0 & 0 & 1 & 0 & 0 & 0 & 0 & + & - & + & & \\
\hline Pilularia minuta & 1 & 0 & 0 & 0 & 0 & 0 & 0 & + & - & - & & 10 \\
\hline Pteridium aquilinum & 3 & 3 & 2 & 2 & 0 & 0 & 3 & + & + & + & $\mathrm{t}$ & \\
\hline Thelypteris palustris & 0 & 0 & 1 & 0 & 0 & 0 & 1 & + & + & + & $\mathrm{m}$ & \\
\hline Asplenium trichomanes quadrivalens & 2 & 3 & 1 & 3 & 0 & 0 & 3 & + & - & + & $\mathrm{m}$ & \\
\hline Asplenium billotii & 3 & 3 & 2 & 3 & 0 & 0 & 3 & + & + & + & $\mathrm{m}$ & \\
\hline splenium onopteris & 2 & 3 & 1 & 2 & 0 & 0 & 2 & + & + & + & $\mathrm{m}$ & \\
\hline Asplenium adiantum-nigrum & 1 & 2 & 1 & 2 & 0 & 0 & 1 & + & - & + & $\mathrm{m}$ & \\
\hline Asplenium septentrionale & 1 & 0 & 0 & 1 & 0 & 0 & 0 & + & + & + & $\mathrm{m}$ & \\
\hline Asplenium ruta-muraria & 1 & 0 & 1 & 0 & 0 & 0 & 0 & + & - & + & $\mathrm{m}$ & \\
\hline Phyllitis scolopendrium & 0 & 0 & 0 & 0 & 0 & 1 & 1 & + & + & + & $\mathrm{m}$ & \\
\hline Ceterach officinarum & 1 & 3 & 1 & 2 & 0 & 1 & 3 & + & - & + & $\mathrm{m}$ & \\
\hline Cystopteris fragilis fragilis & 1 & 2 & 1 & 2 & 0 & 0 & 2 & + & - & + & $\mathrm{m}$ & \\
\hline Cystopteris dickieana & 2 & 2 & 1 & 2 & 0 & 0 & 1 & + & - & + & $\mathrm{m}$ & \\
\hline Cystopteris viridula & 0 . & 1 & 1 & 0 & 0 & 0 & 1 & + & + & + & $\mathrm{m}$ & \\
\hline Athyrium filix-femina & 3 & 2 & 1 & 2 & 0 & 0 & 2 & + & + & + & $\mathrm{m}$ & \\
\hline Athyrium distentifolium & 1 & 0 & 0 & 0 & 0 & 0 & 0 & - & - & + & $\mathrm{m}$ & \\
\hline Dryopteris filix-mas & 3 & 3 & 1 & 3 & 0 & 0 & 1 & + & - & + & $\mathrm{m}$ & \\
\hline Dryopteris affinis affinis & 2 & 0 & 1 & 1 & 0 & 0 & 1 & + & + & + & $\mathrm{m}$ & \\
\hline Dryopteris affinis borreri & 1 & 1 & 1 & 1 & 0 & 0 & 1 & + & + & + & $\mathrm{m}$ & \\
\hline Dryopteris oreades & 1 & 0 & 0 & 1 & 0 & 0 & 0 & - & - & + & $\mathrm{m}$ & \\
\hline Dryopteris carthusiana & 1 & 1 & 0 & 0 & 0 & 0 & 0 & - & - & + & $\mathrm{m}$ & \\
\hline Dryopteris expansa & 1 & 0 & 0 & 0 & 0 & 0 & 0 & - & - & + & $\mathrm{m}$ & \\
\hline Dryopteris dilatata & 1 & 0 & 1 & 1 & 0 & 0 & 1 & + & + & + & $\mathrm{m}$ & \\
\hline Polystichum setiferum & 1 & 1 & 1 & 2 & 0 & 0 & 1 & + & + & + & $\mathrm{m}$ & \\
\hline Polystichum aculeatum & 1 & 0 & 0 & 0 & 0 & 0 & 0 & + & - & + & $\mathrm{m}$ & \\
\hline Blechnum spicant & 3 & 1 & 1 & 1 & 0 & 0 & 1 & + & + & + & $\mathrm{m}$ & \\
\hline Azolla caroliniana & 0 & 1 & 1 & 0 & 0 & 0 & 1 & + & - & + & & \\
\hline Azolla filiculoides & 0 & 1 & 1 & 0 & 0 & 0 & 1 & + & + & + & & \\
\hline $\begin{array}{l}\text { TOTAL: } \\
\text { PROVINCIAS Y SECTORES }\end{array}$ & 42 & 35 & $\begin{array}{l}42 \\
55\end{array}$ & 28 & 3 & 5 & 44 & 52 & 27 & 53 & & \\
\hline
\end{tabular}

Tabla 1. Frecuencia de aparición de los taxones en cada provincia y sector corológico, y otros datos de interés (Ver metodología). Frequency of each taxa at every chorological division, and other interesting data (See methodology). 


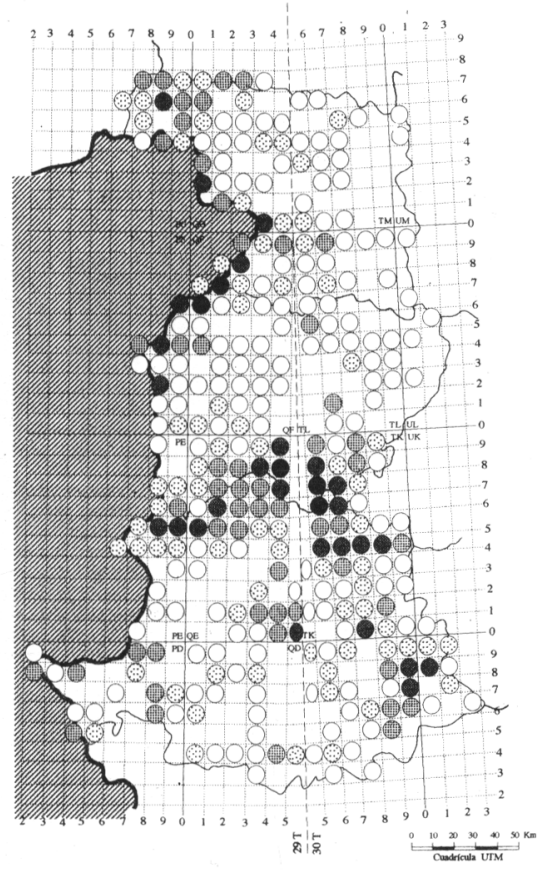

Figura 2. Riqueza de taxones en cuadrículas U.T.M. de $10 \times 10$ para el CW hispano, según los intervalos; O : 1-3; O : 4-7; О:8-12; $: 13-18 ; \bigcirc: 19-25$. Pteridofloristic richness at each 10 x10 U.T.M. square in CW Spain. Richness intervals.

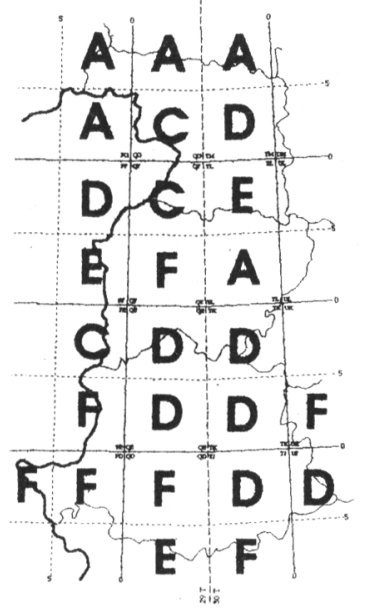

Figura 3. Rangos del índice $\mathrm{m} / \mathrm{t}$ para cada cuadrícula U.T.M. de 50x50 para el CW hispano. Rank values for $\mathrm{m} / \mathrm{t}$ index at each $50 \times 50$ U.T.M. square in $\mathrm{CW}$ Spain. de taxones que crecen en el sector CastellanoDuriense, tan solo cinco, probablemente debido a que se trata de una zona bastante llana ampliamente dedicada a cultivos, por lo que se ven muy reducidos los hábitats que pueden ocupar los pteridófitos.

Índice $\mathrm{m} / \mathrm{t}$. Los resultados del índice para cuadrículas U.T.M. 50x50 se recogen el mapa de la figura 3. Se observan variaciones con los obtenidos en trabajos anteriores (fig. 4), que se basaron en menor cantidad de datos para el CW hispano.

Los mayores valores del índice se encuentran para aquellos lugares en que coinciden condiciones de mayor altitud y menor aridez, o que están situados más al norte del territorio.

Como ejemplo gráfico de esa variación se ha realizado la figura 5. En ella se han representado en ordenadas los valores del índice $\mathrm{m} / \mathrm{t}$, y en abscisas una banda de variación latitudinal (la correspondiente a las cuadrículas U.T.M. de 50x50 QG 1 y siguientes). El valor del índice decrece a medida que nos dirigimos

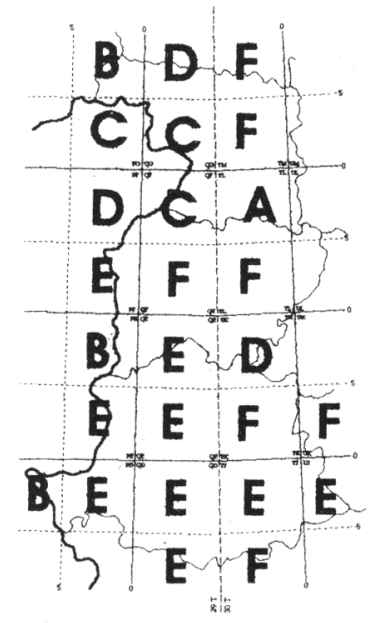

Figura 4. Rangos del índice $\mathrm{m} / \mathrm{t}$ para cada cuadrícula U.T.M. de 50x50, según lo reseñado en Pichi Sermolli et al. (1988). Rank values for $\mathrm{m} / \mathrm{t}$ index at every $50 \times 50$ U.T.M. square, as it was found by Pichi Sermolli \& al. (1988). 


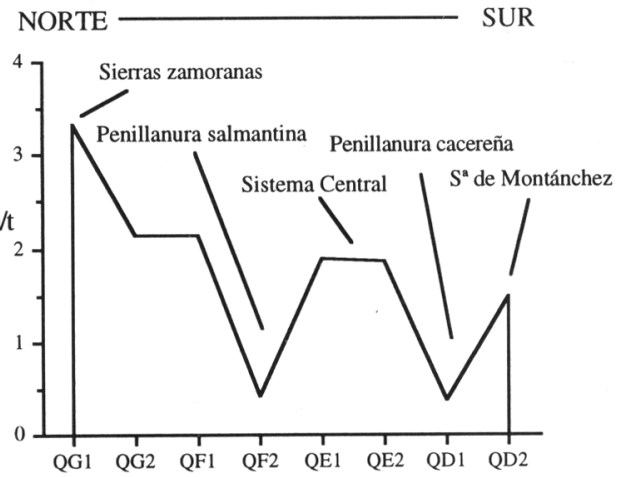

Figura 5. Representación de los valores que toma el índice $\mathrm{m} / \mathrm{t}$ para la banda de variación latitudinal correspondiente a las cuadrículas QG1 a QD2 del retículo U.T.M. de 50x50. Graphical representation of the values adopted by $\mathrm{m} / \mathrm{t}$ index at the latitudinal variation band corresponding to $Q G 1-Q D 250 \times 50$ U.T.M. squares.

\begin{tabular}{lrrl}
\hline SECTORES & $\mathrm{m}$ & $\mathrm{t}$ & $\mathrm{m} / \mathrm{t}$ \\
\hline OS & 23 & 8 & 2,87 \\
LD & 16 & 11 & 1,45 \\
S & 20 & 10 & 2 \\
BG & 18 & 7 & 2,57 \\
CD & 2 & 1 & 2 \\
TT & 20 & 12 & 1,67 \\
PROVINCIAS & & & \\
CMM & 2 & 1 & 2 \\
CIL & 26 & 13 & 2 \\
LE & 20 & 12 & 1,67 \\
\hline
\end{tabular}

Tabla 2. Número de taxones con esporas monoletas o triletas y valores del índice $\mathrm{m} / \mathrm{t}$ en cada provincia y sector corológico del CW hispano. Number of taxa with monolete or trilete spores, and values for $\mathrm{m} / \mathrm{t}$ index at each chorological division in $\mathrm{CW}$ Spain.

hacia el sur, desde las sierras y zonas altas de la provincia de Zamora hasta la penillanura salmantina, tendencia que se interrumpe en las U.T.M. que coinciden con el Sistema Central, donde se combinan los efectos de aumento de humedad y de altitud, de modo que se superponen y enmascaran el efecto que sobre el índice tiene la disminución latitudinal. Al sur de la Sierra de Gata, la aridez y menor altitud de la penillanura cacereña, vuelve a

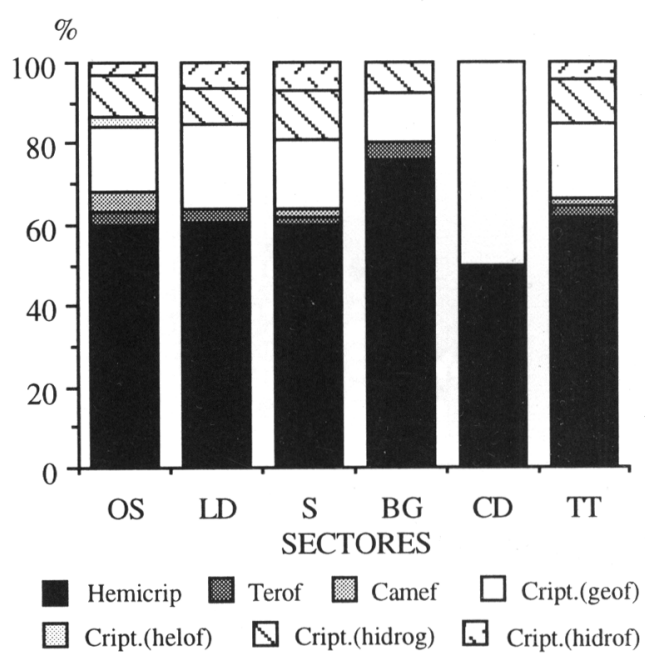

Figura 6. Espectro de formas biológicas de pteridófitos por sectores corológicos. Biological types spectrum for each chorological sector.

provocar una disminución del valor del índice, mas de nuevo la tendencia decreciente se ve rota, aunque de modo menos acusado, al alcanzar la Sierra de Montánchez.

Se corroboran así las conclusiones a que llegó Itô $(1972,1978)$.

Por sectores corológicos, y tal como se observa en la tabla 2, se pueden apreciar las mismas tendencias: Orensano-Sanabrense y Bejarano-Gredense, territorios montañosos y húmedos, acogen mayoritariamente taxones monolaesurados.

Espectro biológico. En la figura 6 se representan las proporciones con que aparecen las diferentes formas o tipos biológicos según la clasificación de Raunkjaer, en lo que a flora pteridofítica se refiere, para cada sector corológico.

El tipo biológico más frecuente en el $\mathrm{CW}$ hispano es el hemicriptófito (el más extendido entre los helechos), tras el que se sitúan los criptófitos en sentido amplio, y entre éstos especialmente el tipo geófito. Los terófitos están representados exclusivamente por 
Anogramma leptophylla .

Solamente dos de los tipos biológicos se encuentran en todos los sectores: el hemicriptófito y el criptófito, siendo el primero especialmente abundante en los sectores más montañosos.

Es de destacar que el tipo criptófito helófito (Equisetum palustre y E. fluviatile) únicamente es conocido del sector OrensanoSanabrense, y que el caméfito (Selaginella denticulata y Lycopodiella inundata) sólo aparece en tres de los sectores: OrensanoSanabrense, Salmantino y Toledano-Tagano.

Espectro edafológico. En este apartado consideramos el comportamiento de los taxones respecto al sustrato, sin incluir a las dos especies del género Azolla dado el carácter hidrofítico de las mismas.

Como cabía esperar, teniendo en cuenta que las tres provincias están situadas dentro de la llamada «España ácida», la gran mayoría de los taxones son acidófilos, aunque es de destacar el elevado número de los que son indiferentes al sustrato.

En la figura 7 representamos por sectores

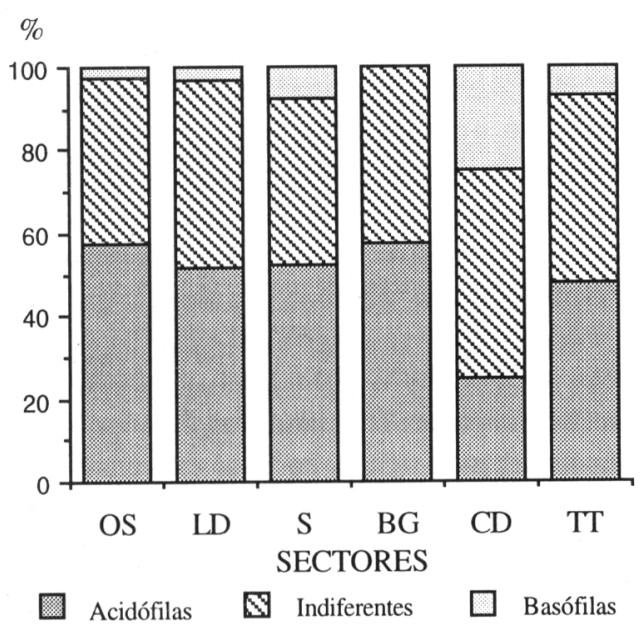

Figura 7. Representación de afinidades edáficas en cada sector corológico. Edaphical affinities representation for each chorological sector. los porcentajes de aparición de los taxones según sus afinidades edáficas.

Solamente hay cuatro taxones basófilos entre los 58 encontrados en la zona de estudio: Cheilanthes acrostica, Phyllitis scolopendrium, Asplenium ruta-muraria y Adiantum capillus-veneris. Estos crecen en ocasiones en argamasas de construcciones humanas, por lo que su presencia dentro de un entorno general con suelos de carácter ácido es explicable (como ocurre con Phyllitis scolopendrium y Asplenium ruta-muraria). En el resto de los casos, dichos taxones, se desarrollan sobre afloramientos de rocas básicas en distintos puntos de las tres provincias.

ISRP: Índice de similitud con las regiones próximas. Se ha encontrado que el carácter de la pteridoflora del CW hispano es eminentemente mediterráneo como cabía esperar, si bien comparte más elementos con la región Eurosiberiana que con la Macaronésica. Sin embargo sería de destacar, la tendencia marcadamente macaronésica del sector Lusitano-Duriense. (Tab. 3).

Similitud entre provincias y sectores. A nivel provincial (tab. 4) se encuentra la mayor semejanza entre la Carpetano-Ibérico-Leonesa y la Luso-Extremadurense (que tienen en común un elevado número de taxones acidófilos o de apetencias oceánicas), mientras que la Castellano-Maestrazgo-Manchega se aparta más de ellas (a pesar de su escasa extensión en la zona, el resultado es lógico por el diferente sustrato y la mayor continentalidad).

Si descendemos al rango sectorial (tab. 5), el mayor parecido se da entre ToledanoTagano y Salmantino, y entre el primero y el Lusitano-Duriense. Aparte del CastellanoDuriense, que por sus características edáficas y bioclimáticas se aleja sistemáticamente de los demás, se observa mínima similitud en cuanto a composición pteridoflorística entre el 


\begin{tabular}{lccccccccc}
\hline $\begin{array}{l}\text { PROVINCIAS } \\
\text { SECTORES }\end{array}$ & GEN & $\begin{array}{c}\text { CMM } \\
\text { CD }\end{array}$ & $\begin{array}{c}\text { LE } \\
\text { TT }\end{array}$ & $\begin{array}{c}\text { CIL } \\
-\end{array}$ & BG & C & LD & OS & L \\
\hline MED & \multirow{2}{*}{66.67} & 8 & 62,85 & 64,90 & 41,93 & 59,42 & 51,90 & 52,17 & 6,06 \\
MAC & 45.71 & 6,97 & 46,03 & 43,79 & 30,90 & 41,93 & 38,31 & 37,1 & 4,70 \\
EUR & 50.26 & 4,28 & 40 & 48,16 & 30,48 & 40,44 & 32,74 & 40,44 & 2,87 \\
\hline
\end{tabular}

Tabla 3. Valores que adopta el ISRP respecto de las Regiones Mediterránea (MED), Macaronésica (MAC) y Eurosiberiana (EUR), para cada una de las provincias y sectores corológicos considerados, y carácter general de la pteridoflora del área de estudio (GEN). ISRP values as concerning to Mediterranean (MED), Macaronesian (MAC) and Eurosiberian (EUR) Regions for each chorological province or sector considered, and general tendency of pteridological flora within CW Spanish area (GEN).

\begin{tabular}{lccc}
\hline \multicolumn{1}{c}{$\%$} & C-I-L & L-E & C-M-M \\
\hline C-I-L & - & & \\
L-E & 82,82 & - & \\
C-M-M & 10,16 & 16,66 & - \\
\hline
\end{tabular}

Tabla 4. Similitud en $\%$ entre las diferentes provincias corológicas representadas en el CW de España. Percentage of similarity between chorological provinces in CW Spain.

Bejarano-Gredense y el Lusitano-Duriense, probablemente por las diferencias altitudinales y bioclimáticas tan acusadas que existen entre ellos.

Indicadores fitogeográficos para el CW hispano. En un análisis minucioso de las áreas de distribución de los taxones (figs. 8 y 9), se observa que algunos de ellos pueden ser tomados en el CW hispano (las consideraciones y conclusiones de este apartado van siempre referidas a nuestro área de trabajo), como indicadores fitogeográficos de las unidades corológicas propuestas por Rivas Martínez (l.c.). De tal modo, en cuanto a provincias corológicas distinguimos pteridófitos tales como: Lycopodiella inundata y Ophioglossum azoricum diferenciales de la provincia Carpetano-Ibérico-Leonesa, y Polypodium vulgare, frecuente en esa provincia y con una sola localidad conocida en la provincia LusoExtremadurense; Selaginella denticulata y Cheilanthes acrostica diferenciales de la Luso-
Extremadurense, y Ophioglossum lusitanicum, muy abundante en la depresión extremeña y con tan sólo tres localidades conocidas dentro de la provincia Carpetano-Ibérico-Leonesa (aunque se trata de enclaves con características ecológicas muy similares a las de la LusoExtremadurense).

El área de la provincia CastellanoMaestrazgo-Manchega representada en el CW hispano, en la que predominan los sustratos básicos, se diferenciaría de las otras dos por la ausencia de numerosos taxones. Así, faltan en ella muchos taxones que son frecuentes en las otras dos, tales como Isoetes sp.pl., Pteridium aquilinum y Asplenium billotii, todos ellos marcadamente acidófilos, y Anogramma leptophylla indiferente al sustrato.

La elevada similitud que existe entre los sectores Bejarano-Gredense y OrensanoSanabrense tiene interés especial por la característica distribución disyunta de dos taxones orófilos en su territorio: tanto Criptogramma crispa como Dryopteris oreades son exclusivos de ellos en el área centro occidental hispana. El mismo caso ocurre entre los sectores Toledano-Tagano y LusitanoDuriense, muy afines entre sí en cuanto a su composición pteridoflorística, con los taxones Cheilanthes maderensis, Cosentinia vellea, Cystopteris viridula y Polypodium cambricum subsp. cambricum. Este tipo de disyunciones se explican principalmente por las características bioclimáticas de los territorios implicados. 


\begin{tabular}{lcccccc}
\hline$\%$ & C-D & B-G & S & L-D & O-S & T-T \\
\hline C-D & - & & & & & \\
B-G & 12,5 & - & & & & \\
S & 13,04 & 71,42 & - & & & \\
L-D & 15,38 & 66,67 & 83,11 & - & & \\
O-S & 13,04 & 80 & 76,19 & 67,53 & - & \\
T-T & 16,67 & 69,44 & 90,69 & 81,01 & 69,76 & - \\
\hline
\end{tabular}

Tabla 5. Similitud en $\%$ entre los diferentes sectores corológicos representados en el CW de España. Percentage of similarity between chorological sectors in CW Spain.

Análisis histórico. En el CW hispano dominan los taxones que alcanzaron el Mediterráneo antes o durante el Terciario frente a los que no lo hicieron hasta el Cuaternario, si bien la presencia de taxones de este último grupo se hace más notable (fig. 10) hacia zonas montanas (sectores Orensano-Sanabrense y BejaranoGredense) que debieron ser las más afectadas por las glaciaciones. Por el contrario, los

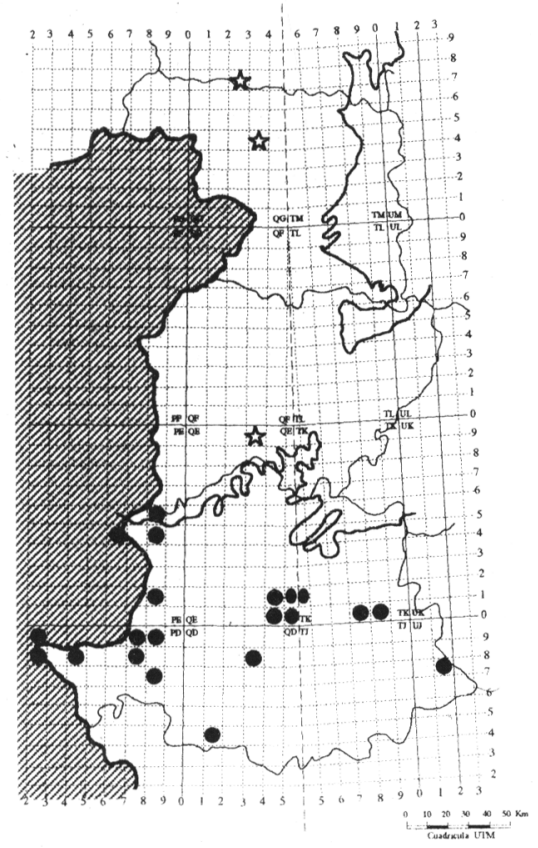

Figura 8. Distribución de Lycopodiella inundata (.) y Selaginella denticulata (.) en el CW hispano. Distribution in CW Spain. sectores Lusitano-Duriense y ToledanoTagano, aparte del Castellano-Duriense, fueron los que menos sufrieron dicha influencia.

Es de destacar la presencia de una especie endémica del Mediterráneo: Pilularia minuta, hallada recientemente (Aedo et al., 1993) en el territorio Orensano-Sanabrense, y de dos relictos Macaronésicos: Ophioglossum azoricum y Cystopteris viridula.

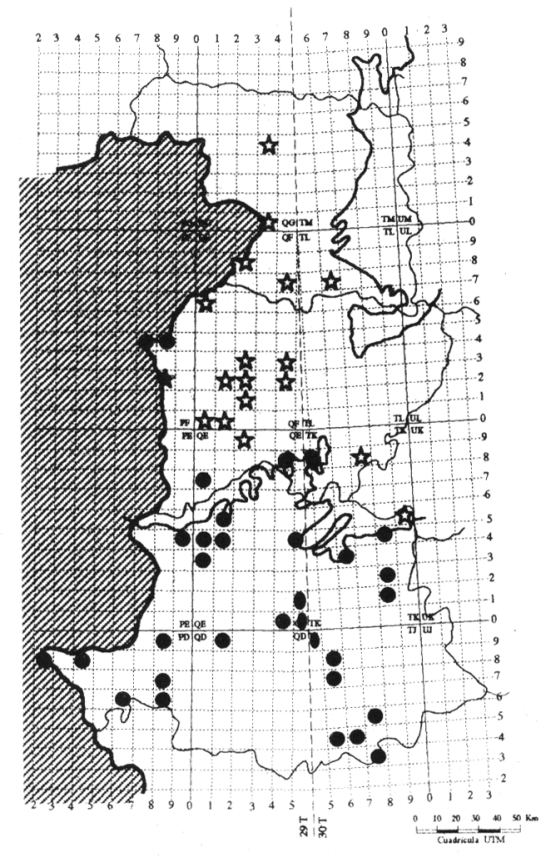

Figura 9. Distribución de Ophioglossum azoricum (1) y Ophioglossum lusitanicum (.) en el CW hispano. Ophioglossum azoricum K. Presl. and Ophioglossum lusitanicum L. distribution in $C W$ Spain. 


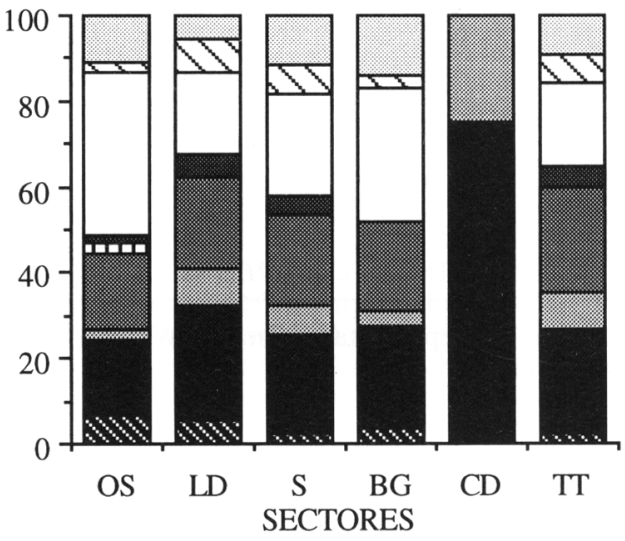

$\mathbf{X}$ Circ.T.(1) Het.T.(2) Med.Pr.(3) Med.Or.T(4)

网 Submed. T.(5) D End.Med.T.(6) Rel.Mac.(7)

$\square$ Circ.C.(8) $\square$ Het.C.(9) $\square$ Med.Pr.C.(10)

Figura 10. Representación de los tipos biogeográficos en cada sector corológico. Biogeographical types representation at each chorological sector.

\section{BIBLIOGRAFÍA}

AEDO, C., J.J. ALDASORO, J.L. DÍAZ ALONSO, J.M. ARGÜELLES, J.M. GONZÁLEZ DEL VALLE, C. HERRÁ, M. LAÍNZ, G. MORENO MORAL, J. PATALLO y O. SÁNCHEZ PEDRAJA -1993- Contribuciones al conocimiento de la flora cantábrica. Fontqueria, 36: 349-374.

CATALÁN, P. e I. AIZPURU -1988- Atlas de los pteridófitos de Navarra. Munibe, Ciencias Naturales, 40: 99-116.

DÍAZ GONZÁLEZ, T.E. y PUENTE GARCÍA, E. 1985- Avance sobre la pteridoflora leonesa. Anales Biol. Murcia, 1: 267-299.

HERRERO BORGOÑóN, J.J., A.M. IBARS, J. IRANZO, M. VILAR, A. PINA, J.C. GARCÍA VERDUGO y A.E. SALVO -1989- Ensayo biogeográfico de la pteridoflora valenciana. Act. Bot. Malacitana, 14: 81-84.

ITÔ, H. -1972- Distribution of monolete and trilete ferns in eastern Asia and northern Oceania. $J$. Jap. Bot., 47: 321-326.

ITÔ, H. -1978- Distribution of two spore patterns in the fern floras of the world. (A preliminary survey). J. Jap. Bot., 53: 164-171.

PICHI SERMOLLI, R.E.G. -1979- A survey of the pteridological flora of the Mediterranean Region. Webbia, 34: 175 - 242.

PICHI SERMOLLI, R.E.G., L. ESPAÑA y A.E. SALVO -1988- El valor biogeográfico de la pteridoflora ibérica. Lazaroa, 10: 187 - 205.

RIVAS MARTÍNEZ, S. -1987-Memoria del mapa de las Series de Vegetación de España. ICONA, Madrid.

SALVO, A.E. y B. CABEZUDO -1984a- Lista comentada de los pteridófitos de Andalucía. Act. Bot. Malacit., 9: 133-146.

SALVO, A.E. y B. CABEZUDO -1984b- Bases para la utilización de los pteridófitos en el establecimiento de unidades corológicas, I. Andalucía. Anales de Biología (Murcia), I: 309316.

SALVO, A.E., B. CABEZUDO y L. ESPAÑA 1984- Atlas de la Pteridoflora Ibérica y Balear. Act. Bot. Malacit., 9:105-128.

SALVO, A.E. y J.C. GARCÍA VERDUGO -1990Biogeografía numérica en Pteridología. In: J. Rita (Ed.), Taxonomía, Biogeografía y Conservación de los Pteridófitos: 115-149.

SÖRENSEN, T. -1948- A method of stablishing groups of equal amplitude in plant sociology based on similarity of species content. Biol. Skr., 5(4): 1-34.

Aceptado para su publicación en Noviembre de 1994

Dirección de los autores. Departamento de Biología Vegetal (Botánica). Facultad de Biología. Universidad de Salamanca. Campus Miguel de Unamuno. 37007. Salamanca. 